\title{
An Active Non-LTR Retrotransposon With Tandem Structure in the Compact Genome of the Pufferfish Tetraodon nigroviridis
}

\author{
Laurence Bouneau, ${ }^{1,4}$ Cécile Fischer, ${ }^{1,4}$ Catherine Ozouf-Costaz, ${ }^{2}$ \\ Alexander Froschauer, ${ }^{3}$ Olivier Jaillon, ${ }^{1}$ Jean-Pierre Coutanceau, ${ }^{2}$ Cornelia Körting, ${ }^{3}$ \\ Jean Weissenbach, ${ }^{1}$ Alain Bernot, ${ }^{1}$ and Jean-Nicolas Volff ${ }^{3,5}$ \\ ${ }^{1}$ Genoscope/Centre National de Séquençage and CNRS-UMR 8030, F-91057 Evry Cedex 06, France; ${ }^{2}$ Laboratoire \\ d'Ichtyologie and Service de Systématique Moléculaire, CNRS IFR 1541, Muséum National d'Histoire Naturelle, F-75231 \\ Paris Cedex 05, France; ${ }^{3}$ BioFuture Research Group "Evolutionary Fish Genomics", Physiologische Chemie I, Biozentrum, \\ University of Würzburg, D-97074 Würzburg, Germany
}

\begin{abstract}
The fish retrotransposable element Zebulon encodes a reverse transcriptase and a carboxy-terminal restriction enzyme-like endonuclease, and is related phylogenetically to site-specific non-LTR retrotransposons from nematodes. Zebulon was detected in the pufferfishes Tetraodon nigroviridis and Takifugu rubripes, as well as in the zebrafish Danio rerio. Structural analysis suggested that Zebulon, in contrast to most non-LTR retrotransposons, might be able to retrotranspose as a partial tandem array. Zebulon was active relatively recently in the compact genome of $T$. nigroviridis, in which it contributed to the extension of intergenic and intronic sequences, and possibly to the formation of genomic rearrangements. Accumulation of Zebulon together with other retrotransposons was observed in some heterochromatic chromosomal regions of the genome of $T$. nigroviridis that might serve as reservoirs for active elements. Hence, pufferfish compact genomes are not evolutionarily inert and contain active retrotransposons, suggesting the presence of mechanisms allowing accumulation of retrotransposable elements in heterochromatin, but minimizing their impact on euchromatic regions. Homologous recombination between partial tandem sequences eliminating active copies of Zebulon and reducing the size of insertions in intronic and intragenic regions might represent such a mechanism.
\end{abstract}

[The sequence data from this study have been submitted to GenBank/EMBL under accession nos. AL808032, AY135221, AJ496734, AJ496221, AJ496222, AJ496223, AJ496224, and AJ496225.]

The different classes of autonomous retrotransposable elements with flanking long-terminal repeats (LTRs) are related at both the structural and phylogenetic levels (Xiong and Eickbush 1990). LTRs are of primordial importance for retrotransposition and are involved in transcription initiation and termination, in synthesis of double-stranded DNA from the RNA intermediate, and are bound by the integrase (Boeke and Chapman 1991). Vertebrate retroviruses as well as Ty1/Copia, $T y 3 / G y p s y$, and BEL retrotransposons have LTRs in direct orientation, but inverted repeats and split direct repeats have been observed in the Dirs1 class of retrotransposons (Goodwin and Poulter 2001).

In contrast, the absence of long flanking sequences is characteristic of non-LTR retrotransposons (also called LINEs or autonomous retroposons). These elements are frequently truncated at their $5^{\prime}$ end by incomplete reverse transcription of their mRNA. Several non-LTR retrotransposons, frequently telomeric, are arranged in a head-to-tail fashion, in which neighboring copies are separated by either poly(A) stretches

\footnotetext{
${ }^{4}$ These authors contributed equally to this work.

${ }^{5}$ Corresponding author.

E-MAIL volff@biozentrum.uni-wuerzburg.de; FAX (0) 931-888-

4150.

Article and publication are at http://www.genome.org/cgi/doi/10.1101/ gr.726003. Article published online before print in June 2003.
}

or target repeat oligomers (Danilevskaya et al. 1997; Takahashi et al. 1997; Arkhipova and Morrison 2001). Interestingly, the promoter of the Drosophila telomeric retrotransposon $H e T-A$ is not located at the $5^{\prime}$ end, but at the $3^{\prime}$ end of the element, and drives the transcription of the downstream HeT-A copy in tandem arrays (Danilevskaya et al. 1997). Hence, the 3' region of HeT-A retrotransposons presents some functional analogy with LTRs (Pardue and Debaryshe 2000).

Both LTR and non-LTR retrotransposons encode a reverse transcriptase. In contrast, the enzymes required for the cleavage and integration at new genomic target sites can be very different. Whereas LTR retrotransposons encode either an integrase, related to the transposase of DNA transposons, or a $\lambda$ recombinase-like protein, non-LTR retrotransposons can encode an apurinic/apyrimidinic endonuclease related to cellular DNA repair enzymes, or a restriction enzyme-like (REL) endonuclease (Feng et al. 1996; Malik and Eickbush 1999; Yang et al. 1999; Goodwin and Poulter 2001). Elements not clearly related to either LTR or non-LTR retrotransposons encode an Uri endonuclease that is also found in group I introns (Lyozin et al. 2001; Volff et al. 2001a).

The release of transposable element copy number constraints appears to be a major characteristic of large genomes (Kidwell 2002). Non-LTR retrotransposons, short interspersed nuclear elements (SINEs), and retrovirus-like sequences to- 
gether make up more than $40 \%$ of the first draft of the human genome (International Human Genome Sequencing Consortium 2001). Certain other vertebrate species have much more compact genomes characterized by small intronic and intergenic sequences and a low percentage of repetitive sequences. With $380-400 \mathrm{Mb}$, the genomes of the marine Japanese pufferfish Takifugu rubripes (Fugu) and the freshwater greenspotted pufferfish Tetraodon nigroviridis are about eight times smaller that the human genome. For this reason, both are objects of (almost) completed genome-sequencing projects (Brenner et al. 1993; Crollius et al. 2000; Fischer et al. 2000; Roest Crollius et al. 2000; Aparicio et al. 2002). Although both
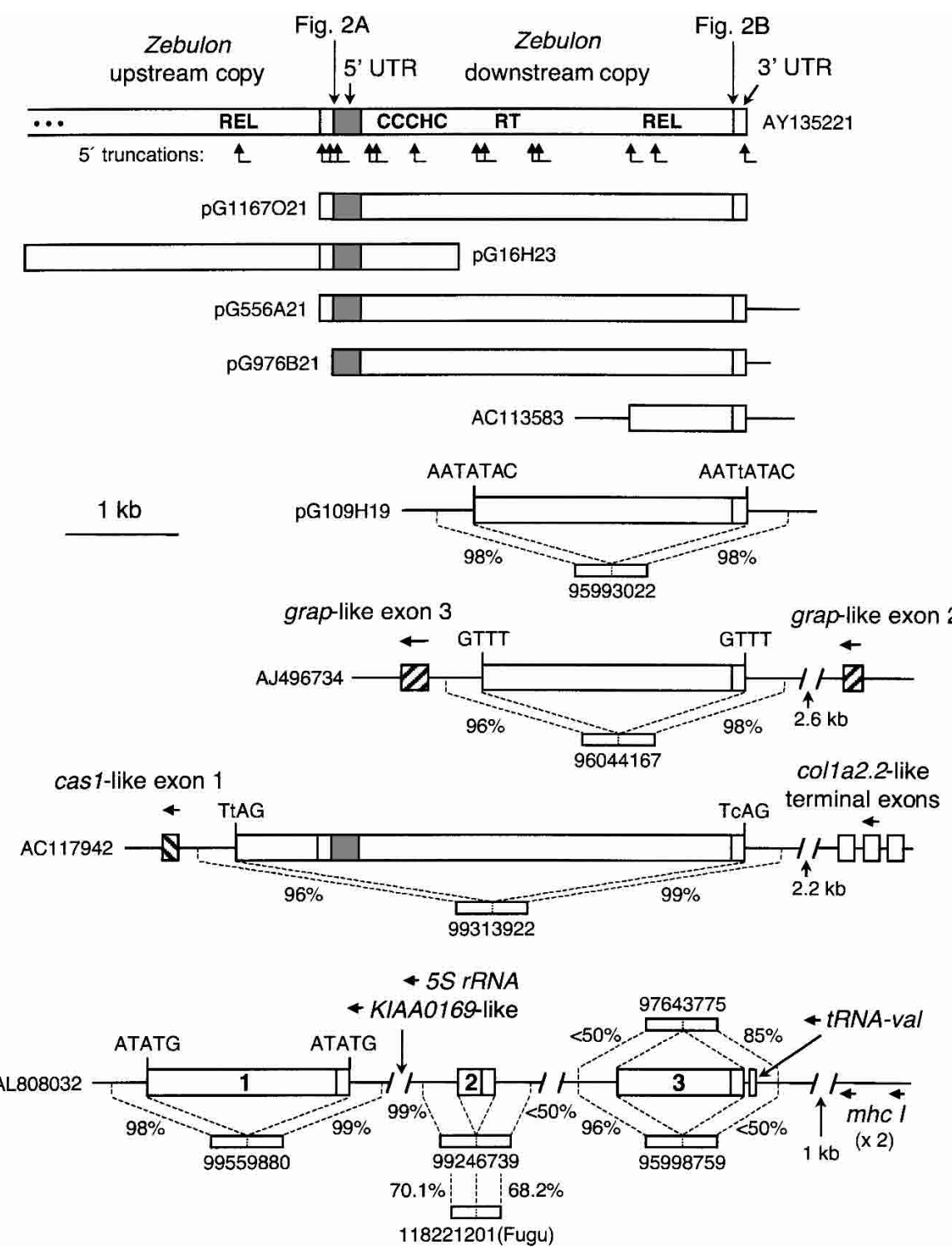

Figure 1 Genomic structure of Zebulon elements in T. nigroviridis. The position of $5^{\prime}$ truncations is shown by broken arrows. Most of them are from single sequences and could therefore not be assigned either to the upstream or to the downstream copy; they were arbitrarily positioned in the downstream copy. Trace sequences (http://www.ncbi.nlm.nih.gov/blast/tracemb.html) reflecting the genomic site before insertion are shown, and their percentage of nucleotide identity with the site sequences directly flanking Zebulon insertions is given. Horizontal arrows show the direction of gene transcription. Putative flanking target site duplications are shown. (RT) Reverse transcriptase; (CCCHC) putative $\mathrm{CXCX}_{3} \mathrm{CX}_{8} \mathrm{HX}_{5} \mathrm{C}$ zinc finger-like domain. pufferfish species are separated by $\sim 20-30$ million years of evolution, the compaction of their genome has been conserved. To understand this phenomenon, it is of primordial transposable elements in pufferfish genomes. Interestingly, and maybe surprisingly, numerous families of retrotransposons have been identified to date in the genome of $T$. rubripes (Aparicio et al. 2002) and/or T. nigroviridis, including Volff et al. 2001b; Goodwin and Poulter 2002), Ty1/Copia (Crollius et al. 2000), BEL (Frame et al. 2001), and Dirs1 classes (Goodwin and Poulter 2001). Non-LTR retrotransposons encoding apurinic-apyrimidinic (Duvernell and Turner 1998; Poulter et al. 1999; Volff et al. 1999, 2000, 2001d) and restriction enzyme-like endonucleases (Volff et al. 2001c), as well as Uri retrotransposons (Volff et al. 2001a) have been identified in pufferfish compact genomes too. We report here the presence of a novel REL endonuclease-encoding non-LTR retrotransposon, called Zebulon, recently active in the genome of $T$. nigroviridis. Interestingly, this non-LTR element frequently displays a tandem structure.

\section{RESULTS}

\section{Zebulon Is a Novel Vertebrate REL Retrotransposon}

In the course of a survey of the retrotransposable element content in the compact genome of the pufferfish $T$. nigroviridis, we identified reverse-transcriptase-encoding sequences with no obvious close relationship to any known vertebrate retroelement. A 3711-bp consensus sequence that we interpreted as the complete version of a novel fish retrotransposon called Zebulon was reconstructed (AY135221; Figs. 1-3). This element was not identified in the recent analysis of the genome of the Japanese pufferfish T. rubripes (Aparicio et al. 2002). Five T. nigroviridis genomic library plasmids containing Zebulon were sequenced completely (pG1167O21, pG16H23, pG556A21, pG976B21, and pG109H19; Fig. 1). Zebulon was also detected in genomic inserts from different bacterial artificial chromosome (BAC) genomic clones from $T$. nigroviridis (AC113583, AJ 496734 , AC117942, and AL808032; Fig. 1). Analysis of the 3' extremity of different Zebulon insertions suggested that this element ended by a short (0-21) poly(A) 
A

Zebulon tandem, upstream copy, 3' UTR

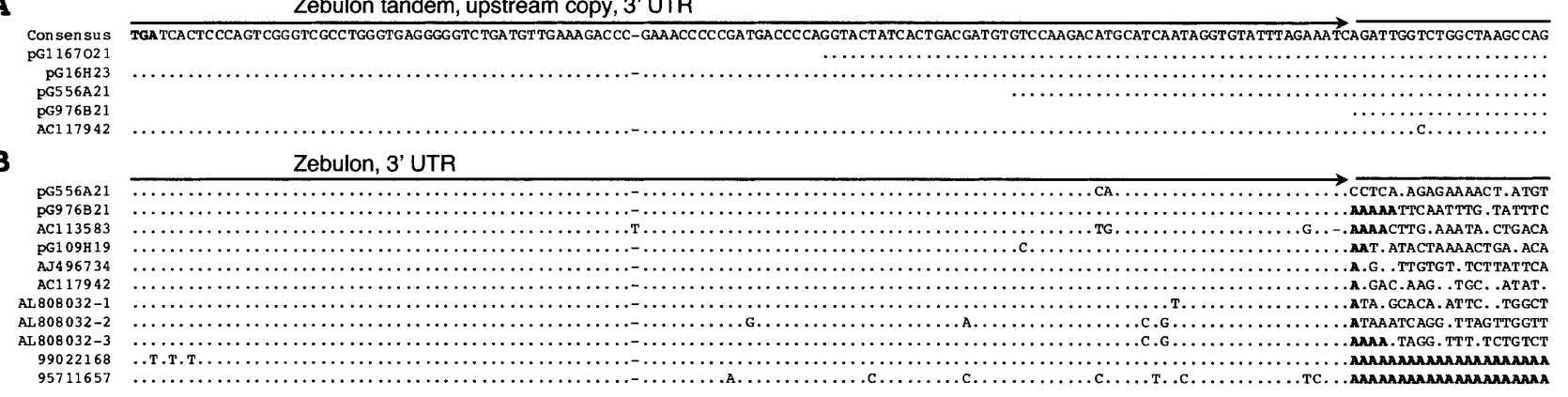

A

Zebulon tandem, downstream copy, 5' UTR

Consensus TGACGTCCAGGAACAGACTGGCTGACGACCACGAATAGAGTGGTGACAGCTTGGATAGACAGCTGACAGCAGGGAAAGACGGCAACCCGCCACGACGGCTACCAACCAGCCTCCATCTTCCGTGAGGAAGAACCCAAAACTTCCTAC

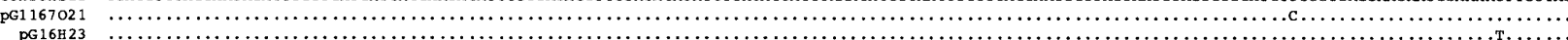
PG556A21

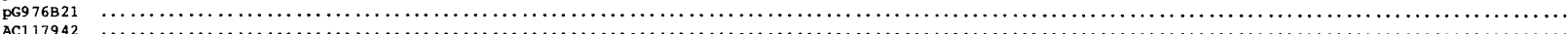

B 3 ' flanking sequence

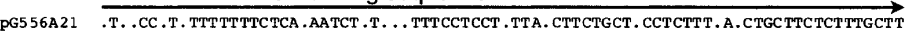
pG976B21 A.G.A.T. . .AC.ATA. AA. AAGACG.GATGAATT.TATCCCAAC TACCA.CA. GG. AGAGAAG. ..CA.TCC. GG. CTT AC1 13583 .T. AC.A.. ATT TA.A.G.. TG. T.T. CAATAAGCACATAAACATGACAGGATC TGTTTGTTT. TTAT TACCCTTT. TG. pG1 09H19 CTGTCCATGTTG TTCTG.GTGAAA......C.A. ... ATCA.CCTGATAT. . AT ..TTCAC.....GATAT. TTTGTT.CA AJ4 96734 .TTTC. .T. ACGTA.A. ACAA.A.T. TGGA. GT. . AC. .T. .CCA. T.TTTG.. .TC. . ATT TTACCCTGCTTT.CT. TTTA

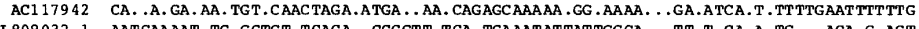
AL.808032-1 AA.AAAA. WA. AL808032-2 .TTACC.TTT.G.A.T. AATAACTGATTGT. ATG. AGA.A.T. GA.AGCA. ... CATTTTGAACTGA. TAT.AA.CT.GA

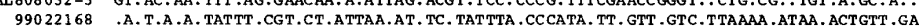

95711657 CAGAAAAAG ..G.ATACAG. GGCTG.TTAG.TGCAG.CT.T. ATCATCT. .AA.GCA.T...T...CGTC.TT.TTTGTTG

A

Zebulon tandem, downstream copy, 5' UTR

Consensus GAAGAGCCCGAAGCAAAGATACCCCCAGGGGAGCCCGAGAGGGGGGGAGAATGAGCTCCC CAAACGGACGGATAACATC

pG1167021

PG16H23

${ }_{\mathrm{pG} 5976 \mathrm{~B} 21}^{\mathrm{PG} 5621}$

Ac117942

(

Figure 2 Sequence comparison between upstream-downstream junctions in tandem arrays $(A)$ and between $3^{\prime}$ ends (B) of Zebulon elements shown in Fig. 1. The part of the tandem array sequences shown extend from the stop codon of the upstream copy to the putative start codon of the downstream copy.

stretch (Fig. 2). We could identify in the T. nigroviridis NCBI trace database, sequences with $>95 \%$ nucleotide identity to both 5' and 3' site sequences flanking some Zebulon insertions (pG109H19, AJ496734, AC117942, and the first copy of AL808032; Fig. 1), but lacking the intervening Zebulon element. These sequences, which are likely to reflect the genomic site before integration, allowed the identification of short target-site duplications flanking Zebulon insertions, AAT(t)ATAC for pG109H19, GTTT for AJ496734, TYAG for AC117942, and ATATG for the first copy of AL808032 (Fig. 1).

Using the reconstructed nucleotide consensus sequence as a query, Zebulon was detected in $\sim 0.2 \%$ of whole-genome shotgun (WGS) sequences from $T$. nigroviridis (4969 of $2,049,513$ sequences with Expect value $\mathrm{E}<10^{-10}$; 4549 sequences with $\mathrm{E}<10^{-20}$; Altschul et al. 1990). A precise copy number could not be estimated from genomic data because of the high variability in copy size, but Southern blot analysis was compatible with the presence of multiple copies of Zebulon in the genome of $T$. nigroviridis (Fig. 4).

\section{Zebulon Is Related to Nematode Site-Specific Non-LTR Retrotransposons}

The unique ORF of Zebulon encodes a putative 1112 aminoacid protein containing a reverse transcriptase domain and a carboxy-terminal restriction enzyme-like endonuclease (REL; Yang et al. 1999) (AY135221; Fig. 3). All amino-acid residues characteristic of REL endonucleases were detected, including a CCHC zinc finger-like domain probably involved in nucleic acid binding (Yang et al. 1999). A second putative (C)CCHC domain is present in the amino-terminal part of the protein upstream from the reverse transcriptase domain, which might correspond to the putative Gag-like domain found in numerous other non-LTR retrotransposons (Fig. 3). Although CCHH zinc finger-like domains are more frequently present at similar positions in REL retrotransposons (see Malik and Eickbush 2000), a CCHC domain was also reported in several elements from trypanosomes (for review, see Gabriel et al. 1990).

Phylogenetic analysis using the reverse transcriptase domain (Malik et al. 1999) supported a relationship between Zebulon and the non-LTR retrotransposon NeSL-1 from the nematode Caenorhabditis elegans (Malik and Eickbush 2000) (data not shown). This was confirmed by using together both the reverse transcriptase and the REL domains as done by Burke et al. (2002) (Fig. 5). The phylogeny obtained confirmed the relationship between NeSL-1 and the $R 4$ clade of REL retrotransposons proposed by Burke et al. (2002). Nevertheless, as the invertebrate elements $R 4$ and Dong are clearly more closely related to Rex6, another fish element, than to Zebulon, $R 4$ and NeSL-1 are likely to correspond to two distinct (sister) clades.

Despite their phylogenetic relationship, Zebulon and NeSL-1 present several essential differences. The cysteine protease domain identified in NeSL-1 (Malik and Eickbush 2000) is apparently absent from Zebulon. In addition, NeSL-1 specifi-

\section{Genome Research}


A

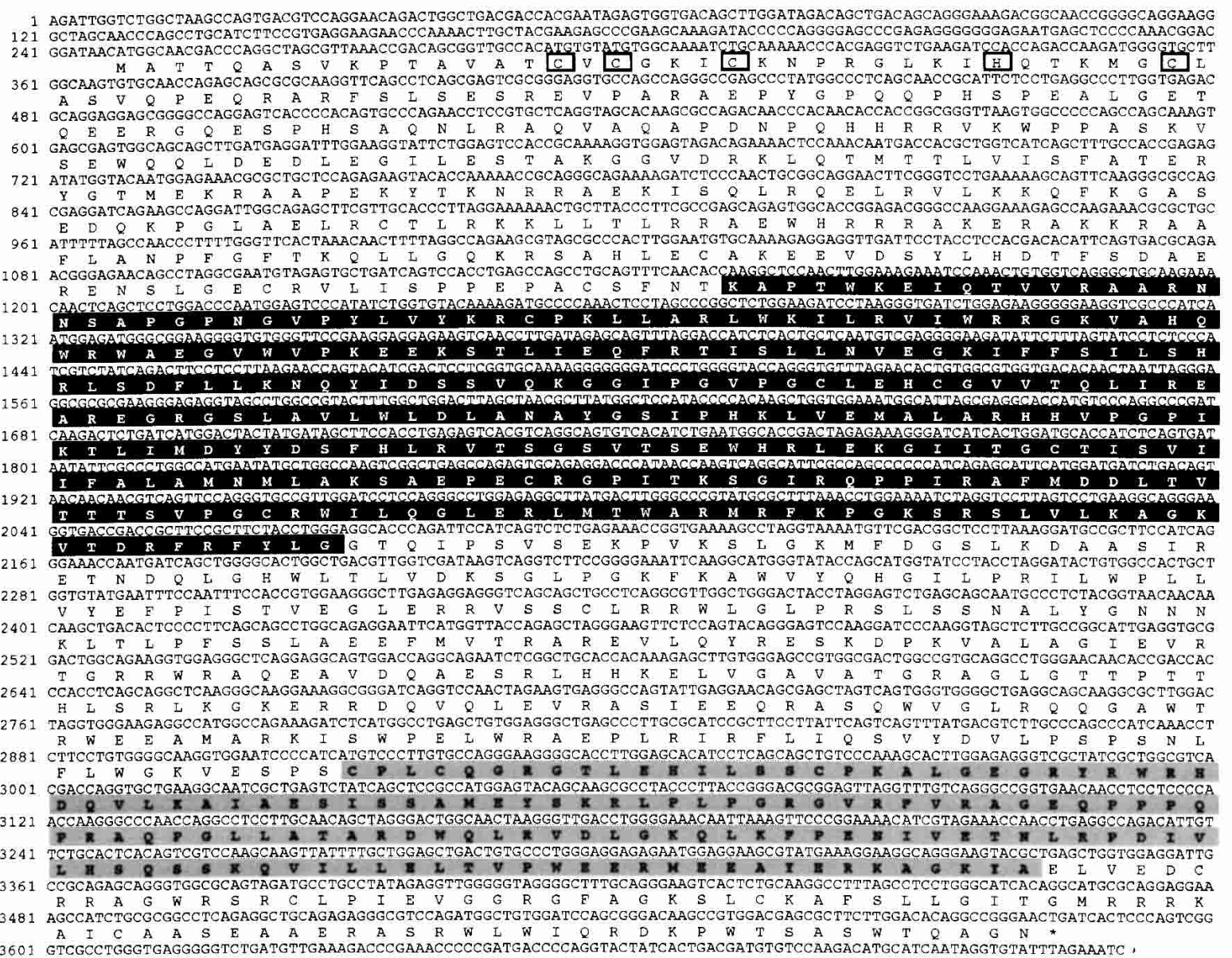

reverse transcriptase

restriction enzyme-like endonuclease

B

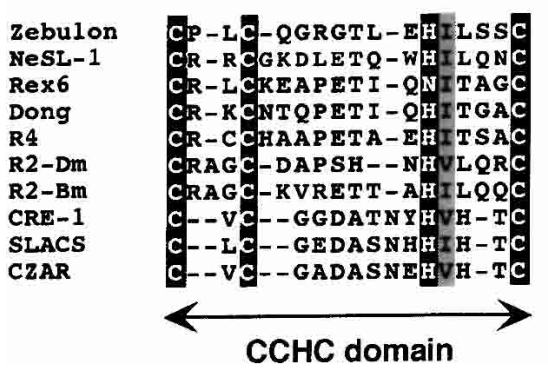

(8) YRWRHDQV (63) P可 (5) LRPDIVLHSQSSKQVILLELTV (11) RKAGKYA (7) ITERHDAV ( 21 ) ERL (5) LRPDICLKSPDEKQIILADVAC (14) AKIDKYE (7) YMERHWQV (27) VEN (19) NQPDIVVVDKEQRKAVVVDVAV (11) EKLEKYQ (7) YTHRHNQL (30) LEN (19) NRPDITLQDKNNKVTYIIDIAV (11) EKMTKYT (7) YVERHDSV (26) SEN (19) RKPDIVVFDLKREKILMFEVSI (11) IKINRYT (6) RVSRHNGV (16) SEQ (8) YKPDIVAF. .RHDSTIVVDAQV (12) SRVEIYN (6) RILRHWK I (16) LEP (8) RKPDIIA..SRDGVGVIVDVQV (12) BKRNKYG (7) RTRRHDGV (16) RDP (16) ARPDLYI..TGSLKPAATDVTI (41) GRTRRYT (7) RQMIRDII (16) TOP (7) RRPDILIAGL..DTYAVTDITV (32) EKERKYS (7) RTTRHDMI (16) MEP (7) RRPDILIVGL..DTYAITDVTV (29) QRRQRYR CCHC domain

REL domain

Figure 3 Consensus sequence of the Zebulon retrotransposon of T. nigroviridis. (A) Complete consensus sequence. Amino-acid residues forming the putative amino-terminal (C)CCHC zinc finger domain are boxed. (B) Restriction enzyme-like domain.

cally inserts into the spliced leader-1 gene of C. elegans. In contrast, inspection of $\sim 20$ different Zebulon $5^{\prime}$ and $3^{\prime}$ extremities could not reveal any target specificity beside a slight preference for T (Fig. 2; data not shown). No obvious similarity could be found between the different duplicated target sequences identified for pG109H19, AJ496734, AC117942, 

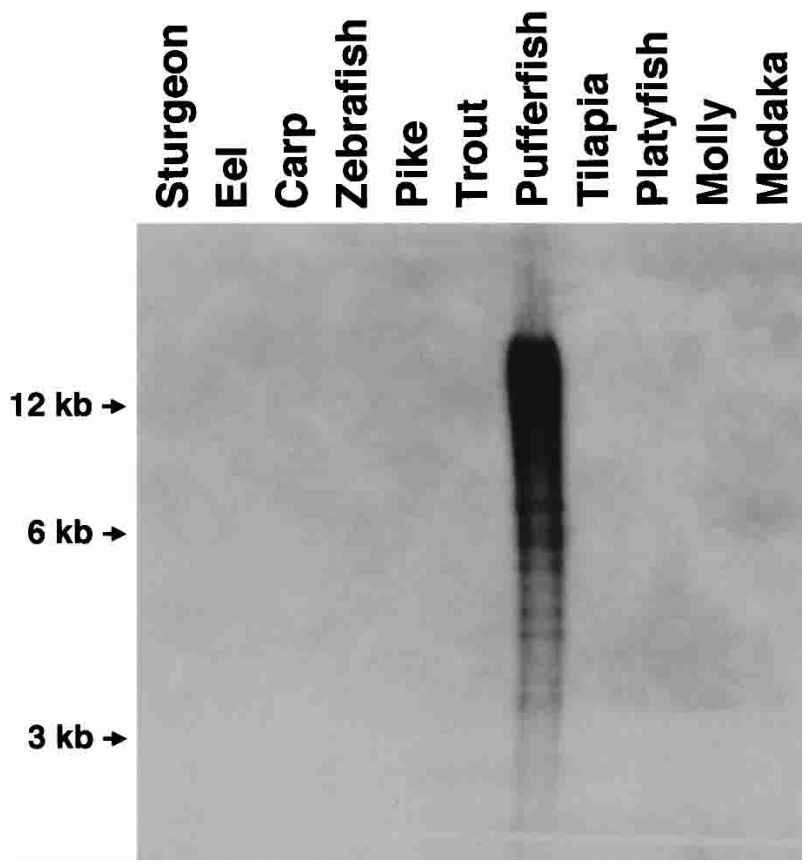

Figure 4 Southern blot analysis of the distribution of Zebulon in fish. Genomic DNA was cut with HindIII (does not cut in Zebulon). The probe used is pG16H23. Origin of fishes is given in Crollius et al. (2000) and Volff et al. (2000).

and the first copy of AL808032 (AATtATAC, GTTT, TYAG, and ATATG, respectively; Fig. 1).

\section{Zebulon Copies With a Tandem Structure}

During the reconstruction of a $T$. nigroviridis consensus element by assembling shotgun genomic sequences, it became evident that the 3711-bp Zebulon unit was flanked frequently on its $5^{\prime}$ side by a sequence corresponding to the $3^{\prime}$ end of the element. This tandem structure was, for example, observed in genomic inserts of BAC clone AC117942 and plasmids pG1167O21, pG16H23, and pG556A21 (Fig. 1). Strikingly, the junction between the 3 ' end of the upstream copy and the 5 ' end of the downstream copy was exactly at the same position in all database tandem sequences analyzed (Fig. 2; data not shown). This was probably not resulting from overrepresentation of a particular tandem element in databases, as identical junctions were found in Zebulon tandem elements corresponding to different insertions (for example, BAC clone AC117942, plasmids pG556A21, and pG16H23, Figs. 1-2; shotgun sequences AL251620 and AL191204 with strongly 5' truncated upstream copy; data not shown). After analysis of the $5^{\prime}$ extremity of copies without 5' duplicated region, no evidence for an alternative structure at the 5' end of Zebulon could be found; all of these copies corresponded to truncated versions of the 3711-bp unit, the position of the truncation varying between different copies (Fig. 1). The upstream copy was also $5^{\prime}$ truncated in tandem arrays, generating a sort of LTR-like structure. The position of the $5^{\prime}$ truncation in the upstream copy was different in different tandem arrays of Zebulon (e.g., pG16H23, AC117942, Fig. 1; AL251620 and AL191204; data not shown). No 5' truncation in the downstream copy was detected when an upstream copy was present.

\section{Zebulon Extends Intronic and Intergenic Regions in the Compact Genome of $T$. nigroviridis}

Zebulon can integrate into intronic and intergenic sequences in the genome of $T$. nigroviridis. One copy of Zebulon (AJ496734, Fig. 1) is integrated only 400 nucleotides away from the third exon of a gene encoding a protein homologous to Grap, an adaptor protein coupling tyrosine kinases to the Ras pathways in human (Q13588). In AC117942, Zebulon is present between two genes, $\sim 400$ nucleotides upstream from the first exon of a gene encoding a product homologous to the human Cas1p O-acetyltransferase (AAL33538) and 2.2 $\mathrm{kb}$ away from the terminal exon of a gene related to the type I collagen $\alpha 2$ chain gene col1a2.2 from chum salmon (BAB79230) (Fig. 1). In AL808032, copy 3 of Zebulon is integrated only $54 \mathrm{bp}$ from a putative tRNA-Val gene, and at the proximity of MHC class I gene duplicates. All of these copies were integrated in an opposite transcriptional orientation compared with the neighboring exons. These observations indicate that Zebulon is a retrotransposon occasionally contributing to the extension of intronic and intergenic regions in the compact pufferfish genome.

\section{Preferential Localization of Zebulon in Some Heterochromatic Regions}

According to Fischer et al (2000), DAPI brightly stains, after denaturation treatment, heterochromatic regions in the chromosomes of $T$. nigroviridis, that is, short arms of subtelocentric chromosomes and pericentromeric regions. These regions correspond mostly to satellite repeats and other kinds of repetitive sequences (Crollius et al. 2000; Dasilva et al. 2002). Zebulon hybridizes mainly in those areas, showing major regions of accumulation in at least five chromosome pairs (Fig. $6 \mathrm{a} 1, \mathrm{a} 2)$. Other weaker signals were usually detected at the end of the arms of subtelocentric chromosomes and in pericentromeric regions. Moreover, when the pG16H23 probe was cohybridized on $T$. nigroviridis chromosomes with a plasmid containing the non-LTR retrotransposon Rex3 (Volff et al. 1999; C. Fischer, L. Bouneau, and C. Ozouf-Costaz, unpubl.), signals were, in most cases, overlapping, particularly for the major signals of Zebulon (Fig. 6b1-b4).

\section{Recent Activity of Zebulon in T. nigroviridis}

Even if some more divergent copies with only $80 \%$ nucleotide identity are present in the T. nigroviridis trace database, comparison of the 11 different Zebulon elements shown in Figure 1 revealed a general high level of nucleotide identity (between $94.1 \%$ and $99.7 \%$, average $97.6 \%$ ). Particularly, the degree of nucleotide identity between the clearly different insertions in genomic sequences AJ496734 and AC117942 was as high as $99.7 \%$. The complete ORF of the downstream copy of AC117942 was intact and its putative translation product displayed only two conservative differences over 1112 aminoacids compared with Zebulon consensus protein sequence. Zebulon in genomic sequence AJ496734 was truncated at its $5^{\prime}$ end (Fig. 1). Nevertheless, the remaining part of the ORF was still intact and its conceptual product showed only one conservative and one nonconservative replacement over $738 \mathrm{ami}-$ noacids. Hence, the very high degree of sequence identity between different Zebulon insertions added to the presence of noncorrupted, possibly functional copies, indicate that this element retrotransposed relatively recently and might be still active in the compact genome of the pufferfish $T$. nigroviridis. 


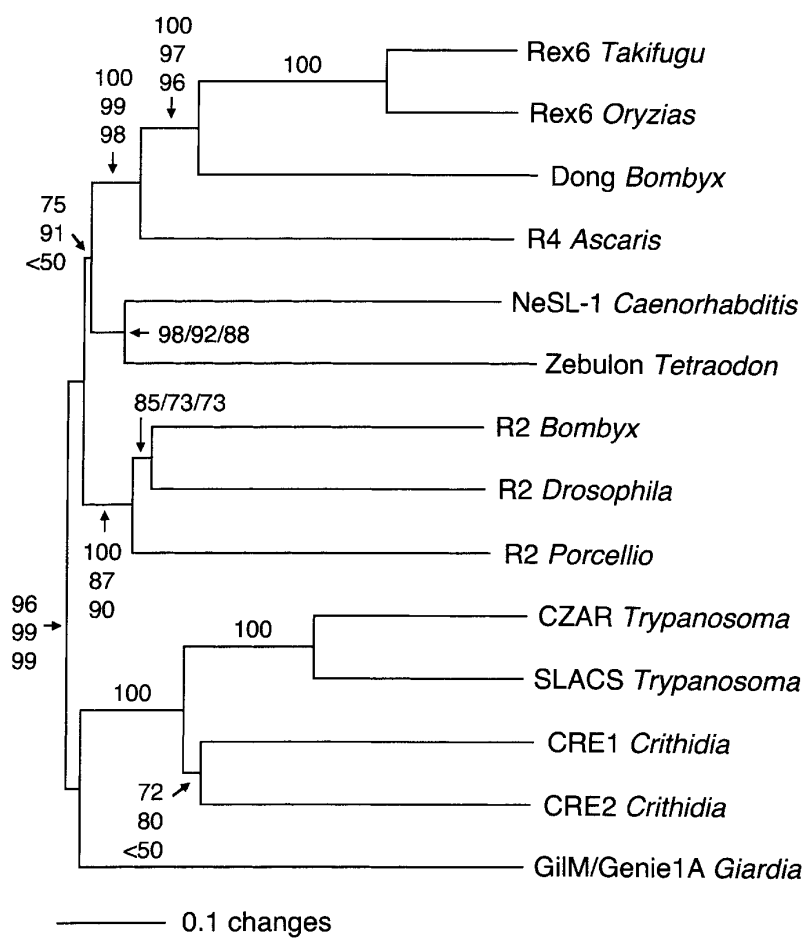

Figure 5 Phylogenetic relationship between Zebulon and the nematode site-specific retrotransposon NeSL-1. Phylogeny was performed using together the reverse transcriptase and REL domains (Burke et al. 2002). The tree (neighbor-joining) is unrooted. Branches with $<50 \%$ support have been collapsed. Bootstrap values using neighbor-joining (1000 replicates, first values) and maximum parsimony analyses (100 replicates, third values), as well as reliability values for maximum likelihood analysis (quartet puzzling, 10,000 puzzling steps, second values) are given. Accession numbers are the same as in Malik et al. (1999) and Burke et al. (2002).

Involvement of Zebulon in Genomic Rearrangements? Using the genomic sequences flanking copies 2 and 3 from BAC clone AL808032 as queries against T. nigroviridis sequence databases, corresponding sequences without Zebulon insertion were identified (e.g., NCBI trace sequences 99246739 and 95998759 for copies 2 and 3, respectively; Fig. 1). Strikingly, these unoccupied sites showed $>95 \%$ nucleotide identity to the $5^{\prime}$ sequence flanking directly the insertions (identity ending exactly at the position of the Zebulon insertion), but no significant identity to the $3^{\prime}$ sequence flanking the insertion, or to any other sequence present in AL808032. A sequence corresponding to $T$. nigroviridis sequence 99246739 and also presenting significant nucleotide identity only to the $5^{\prime}$ sequence flanking insertion 2 in AL808032 was identified in T. rubripes (trace sequence 118221201; Fig. 1). In addition, T. nigroviridis sequence 97643775 presented $85 \%$ nucleotide identity to the 3 ' sequence directly flanking copy 3 in AL808032, but showed no significant identity to the $5^{\prime}$ flanking sequence (Fig. 1). To exclude that the structure observed for copies 2 and 3 in AL808032 was the result of cloning or assembling artifacts having eliminated the intervening sequence between two nonallelic copies of Zebulon, both copies 2 and 3 were amplified by PCR from $T$. nigroviridis genomic DNA using primers matching their $5^{\prime}$ and $3^{\prime}$ flanking sequences. The size of the obtained PCR fragments and their sequence confirmed that the structure of copies 2 and 3 in AL808032 is also found in $T$. nigroviridis genome (data not shown). The structure observed for copy 2 and 3 might have been created by ectopic homologous recombination between two nonallelic copies of Zebulon. For example, recombination between two copies, one inserted in a 95998759-like site and one integrated in a 97643775-like site (Fig. 1), may have generated a hybrid element with flanking sequences originating each from different genomic sites. Hence, Zebulon might be involved in the formation of rearrangements in the genome of $T$. nigroviridis. Alternatively, the structure observed in copy 2 and 3 might be the result of deletions having affected the $5^{\prime}$ genomic sequence flanking Zebulon insertions. Such deletions are associated with the retrotransposition of $L 1$ in transformed human cells (Gilbert et al. 2002; Symer et al. 2002), but retrotransposition-independent deletions having included both the $5^{\prime}$ part of the element and its 5' flanking sequence might generate the same type of structure.

\section{Is Zebulon Fish Specific?}

The distribution of Zebulon was studied by Southern blot analysis and homology searching of sequence databases. Using pG16H23 from T. nigroviridis as a probe in Southern blot hybridization, no significant signal could be detected even under low-stringency conditions in 10 other fish species (Fig. 4; the Japanese pufferfish T. rubripes was not included).

In contrast, Zebulon sequences presenting an average $67.5 \%$ nucleotide identity (from $63.1 \%$ to $74.5 \%$ ) to $T$. nigroviridis elements were identified in $T$. rubripes by database analysis. A short Zebulon element is located $\sim 850$ bp upstream of the second exon of a gene encoding the rho-type GTPaseactivating protein rhoGAPX-1 (AF012274). Zebulon elements are also present in at least 10 of 12,403 WGS scaffolds from the genome draft of $T$. rubripes (http://fugu.hgmp.mrc.ac.uk/; Aparicio et al. 2002), all of them with ORFs corrupted by $5^{\prime}$ truncations, frameshifts, and/or stop codons. Zebulon copies identified in $T$. rubripes presented to each other a level of nucleotide identity ranging from $87.9 \%$ to $96.5 \%$ (94.1\% on average; more divergent sequences with only $\sim 75.0 \%$ identity are also present in the trace database). An almost complete 3.6-kb Zebulon sequence could be reconstructed from different genomic scaffolds and was used as a query against the $T$. rubripes WGS trace database $(1,877,457$ sequences with an average size of 920 nucleotides). A total of 246 sequences ( $0.013 \%$ vs. $0.24 \%$ for $T$. nigroviridis) showed significant nt identity to Zebulon $\left(\mathrm{E}<10^{-10}\right.$, a threshold allowing the detection of copies with $<80 \%$ nucleotide identity). This suggested that the $T$. nigroviridis genome contains 15-20 times more copies of Zebulon than the genome of T. rubripes. This conclusion was not modified by choosing a more stringent threshold $\left(\mathrm{E}<10^{-20} ; 0.0093 \%\right.$ for $T$. rubripes vs. $0.22 \%$ for $T$. nigroviridis).

Zebulon was also detected in the genome of the zebrafish Danio rerio, which diverged from pufferfishes $\sim 150$ million years ago. Elements truncated at their $5^{\prime}$ end and presenting various other kinds of corrupting mutations were identified in at least 10 different database genomic sequences (e.g., AL627164, AL929152, and AL928790). These copies shared from $61.1 \%$ to $67.6 \%$ nucleotide identity (average 63.9\%) with Zebulon elements from $T$. nigroviridis, probably explaining the absence of signal in Southern blot hybridization (Fig. 4). Zebulon copies of zebrafish showed an average $82.2 \%$ nucleotide identity to each other (from $72.2 \%$ to $89.8 \%$ ). $\mathrm{Ze}$ - 

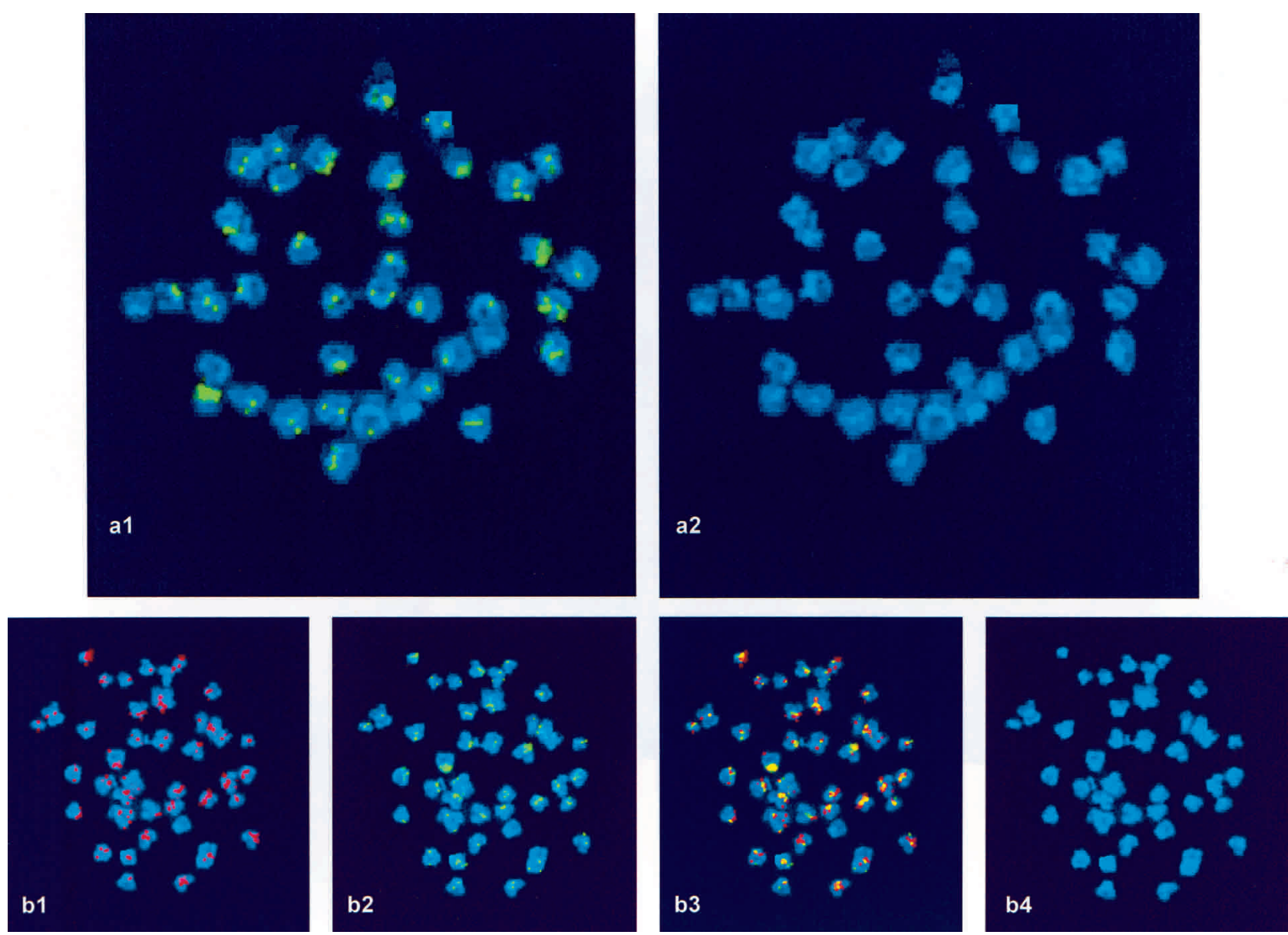

Figure 6 Chromosomal localization of Zebulon in the genome of $T$. nigroviridis by FISH. Weak, scattered spots have been removed by electronic thresholding in order to retain only major regions of accumulation. The genomic areas in which Zebulon preferentially localizes (a1) mostly correspond to heterochromatic, DAPI-positive regions as shown in this over-denaturated metaphase (a2). Double FISH between Zebulon (DIGlabeled $\mathrm{pG} 16 \mathrm{H} 23, b 1)$ and $\operatorname{Rex} 3$ (biotin-labeled, b2), a non-LTR retrotransposon abundant in the genome of $T$. nigroviridis (C. Fischer, L. Bouneau, and C. Ozouf-Costaz, unpubl.) shows superimposed signals (b3) corresponding to common regions of accumulation in DAPI-positive regions (b4).

bulon was also detected in about 90 of 158,689 contigs from the zebrafish WGS assembly 06 (http://www.ensembl.org/ Danio_rerio/blastview). An almost complete 3.5-kb copy of Zebulon was identified in WGS contig z06s014441 and used as a query against the NCBI zebrafish WGS trace database $(11,453,550$ sequences with an average length of 700 nucleotides). The results indicated that $0.005 \%-0.007 \%$ of the zebrafish WGS sequences contained Zebulon (781 sequences with $\mathrm{E}<10^{-10} ; 587$ sequences with $\mathrm{E}<10^{-20}$ ), a value much lower than that obtained for $T$. nigroviridis. We could not establish without ambiguity whether the tandem structure observed in $T$. nigroviridis was also present in T. rubripes and D. rerio.

Zebulon was not detected outside of the fish lineage in the huge amount of sequences present in databases. Particularly, Zebulon was not present within the public draft of the human genome. Hence, if we assume a mode of vertical transmission, Zebulon might have been lost from some vertebrate lineages.

\section{DISCUSSION}

Retrotransposons encoding a restriction enzyme-like endonuclease have been identified originally in insects and other invertebrates (Yang et al. 1999). After the Rex6 element from fish (Volff et al. 2001c), Zebulon is the second retrotransposon of this type to be identified in vertebrates. Zebulon was not detected in the human genome by sequence database analysis. Other instances of retrotransposable elements active in fish, but apparently either absent from mammals or present as inactive molecular fossils, have been reported already (Volff et al. 2001e). These observations suggest a greater diversity of active retrotransposable elements in fish compared with human and probably other mammals. Thinking in terms of competition, the extinction of some families of retrotransposons in the mammalian lineage might have allowed, or alternatively might have been caused by the formidable expansion of both $L 1$ non-LTR retrotransposons and vertebrate endogenous retroviruses (International Human Genome Sequencing Consortium 2001).

Most families of retrotransposable elements described in teleost fish are present in the genome of the pufferfishes $T$. nigroviridis and T. rubripes. Despite the presence of multiple families of retroelements, a strong compaction of the genome (eight times smaller than the human genome) has been maintained for unknown reasons in both pufferfishes since their

\section{Genome Research www.genome.org}


divergence 20-30 millions years ago. Even if exceptional genes exist (Aparicio et al. 2002), small intergenic and intronic regions and a low percentage of repetitive sequences are characteristic of both pufferfish compact genomes. Using Zebulon as an example, we could show that pufferfish genomes contain retrotransposons having been very recently (and probably still) active. Zebulon was apparently more successful in the freshwater pufferfish $T$. nigroviridis than in the Japanese pufferfish T. rubripes or even than in the zebrafish $D$. rerio having an approximately three times larger genome. $Z e$ bulon is a factor contributing to the extension of intergenic and intronic sequences. If there is a selection maintaining genome compaction in pufferfishes, it should act strongly against Zebulon retrotransposition in gene-rich regions.

If we assume a vertical modus of inheritance, putative mechanisms might explain the maintenance of Zebulon activity in the compact genome of T. nigroviridis. As revealed by FISH experiments, Zebulon preferentially concentrates within some heterochromatic regions, generally within short chromosome arms or pericentromeric regions. Very recently, this phenomenon has been also reported for other tandem and dispersed repeat elements in the same fish species (Dasilva et al. 2002). Preferential localization of retrotransposable elements in heterochromatin has been reported frequently in other genomes (Dimitri and Junakovic 1999; Bartolomé et al. 2002), but its significance remains controversial. Generally, heterochromatic retrotransposable elements are defective (for example, see Vaury et al. 1989). On the other hand, such gene-poor heterochromatic regions might serve as reservoirs that are tolerated by the genome, and can maintain active copies of Zebulon (an advantageous role of retrotransposons in heterochromatin has even been proposed, see Dimitri and Junakovic 1999). Interestingly, Zebulon colocalized very frequently in FISH experiments with Rex3, another abundant non-LTR retrotransposon, suggesting the presence of general heterochromatic reservoirs for retrotransposable elements. Nevertheless, the reservoir theory implies that these retrotransposons can use promoters that are active in the generally gene-silencing heterochromatin, as reported for the HeT-A retrotransposon in Drosophila (Danilevskaya et al. 1997; Pardue and Debaryshe 2000).

Which mechanisms might be responsible for the uneven distribution of Zebulon in heterochromatic and euchromatic regions? Zebulon might possess some kind of (non-strict) specialization for heterochromatic regions, as observed for telomeric retrotransposons in some organisms (Danilevskaya et al. 1997; Takahashi et al. 1997; Arkhipova and Morrison 2001). Nevertheless, we could not observe any target sequence specificity for Zebulon, indicating that if a preference is present, it is probably not driven by the primary sequence of the target site. On the other hand, drastic preferential elimination of retrotransposons in euchromatin might occur, maintaining the compaction of gene-rich regions in the pufferfish. This might be particularly achieved by natural selection against individual insertions, against genomic rearrangements mediated by ectopic homologous recombination between non-allelic copies, and/or against retrotransposition itself if it occurs at the cost of the host (Bartolomé et al. 2002; Eickbush and Furano 2002, and references therein).

A possible advantage of Zebulon in the compact genome of the pufferfish is suggested by the observation that this nonLTR retrotransposon frequently displays a partial tandem structure with variable $5^{\prime}$ truncations of the upstream copy. Homologous recombination between the 3 ' ends of both up- stream and downstream copies might lead to the elimination of active elements and reduce the size of Zebulon insertions in a mechanism reminiscent of that generating solo LTRs from LTR retrotransposons and retroviruses. This might minimize the effect of Zebulon on the extension of intergenic and intronic sequences and maintain the number of active copies to a number tolerable by pufferfish euchromatin.

The mechanism of formation of Zebulon tandem arrays remains unknown. Particularly, we do not know whether they are the result of successive events of retrotransposition, or whether the tandem array itself can be retrotransposed. In non-LTR retrotransposons arranged in head-to-tail arrays, the tandem structure is generated by successive events of retrotransposition, and the different units are either separated by poly(A) stretches of different lengths, or by a variable number of copies of the repeated sequence serving as targets (e.g., telomeric repeats). Alternatively, some retrotransposons can create tandem arrays by jumping into themselves, generally at different positions inside of the target element (Higashiyama et al. 1997). In contrast, the identity of the junctions between upstream and downstream copies in different elements suggests that Zebulon tandem arrays might function as a retrotransposition unit. The structure of the tandem array in sequence AC117942 with short flanking sequence duplications is compatible with a single integration event.

The promoter(s) driving the transcription of Zebulon remains to be identified. A promoter located within the upstream copy might be able to promote the transcription of partial tandem arrays, in a manner reminiscent of that reported for the telomeric retrotransposon $\mathrm{HeT}$ - $\mathrm{A}$ in Drosophila (Danilevskaya et al. 1997). Because of the almost impossibility of performing functional analysis in $T$. nigroviridis due to the absence of laboratory strains, cell lines, and transgenesis technology, we are not able at the moment to provide any information about the promoter region(s) driving the transcription of Zebulon.

The use of a $3^{\prime}$ promoter, coupled to variable degrees of $5^{\prime}$ truncation by incomplete reverse transcription, might generate the tandem structures of variable lengths observed in some copies of Zebulon. Alternatively, nonreproducible truncations of the upstream copy in tandem arrays might be due to the use of alternative transcription starts from a same promoter, or to the presence of different promoters in the upstream copy. Finally, tandem arrays might have been generated by the massive transcription of a single tandem fortuitously integrated at the neighborhood of an exogenous strong promoter. Because functional analyses are almost impossible in pufferfish, functional Zebulon elements have now to be identified and characterized in alternative fish model systems to elucidate the mechanism of retrotransposition and the genomic impact of this interesting retroelement.

\section{METHODS}

\section{Plasmids and DNA Manipulation}

T. nigroviridis genomic libraries and sequencing procedures have been described elsewhere (Crollius et al. 2000; Fischer et al. 2002). Zebulon-containing plasmids pG109H19, pG1167O21, pG16H23, pG556A21, and pG976B21 (Fig. 1; AJ496221-AJ496225) were identified by end-sequencing in a plasmid genomic library with average insert size of $4 \mathrm{~kb}$, and sequenced subsequently to completion. Genomic DNA isolation and Southern blot analysis were performed according to standard protocols (Volff et al. 1999, and references therein). 
Southern blot hybridization was performed in 35\% formamide at $42^{\circ} \mathrm{C}$, the filter was washed with $2 \times$ SSC/1\% SDS at $50^{\circ} \mathrm{C}$.

\section{FISH Analysis}

Zebulon-containing plasmids were digoxigenin (DIG) or biotin labeled for FISH analysis by nick translation (Roche). Labeled probes were purified using the Qiaquick PCR purification kit (QIAGEN), ethanol precipitated, and mixed up again in QBIO-gene high-stringency Hybrisol VI at $20 \mathrm{ng} / \mu \mathrm{L}$ each. Probes were hybridized and detected on T. nigroviridis freshly thawed chromosome preparations without any pretreatment, according to the protocol of QBIO-gene for repetitive probes (Crollius et al. 2000). Preparations were counterstained simultaneously, mounted with $1.2 \mathrm{ng} / \mu \mathrm{L}$ DAPI in Antifade (Vector Laboratories), and analyzed using Genus FISH-imaging equipment and software for animal chromosomes (Applied Imaging). For unequivocal chromosome localization of Zebulon, double FISH was performed with two different plasmids (biotin-labeled pG109H19 and DIG-labeled pG16H23), and their correct overlapping was checked. Only results obtained with pG16H23 are shown.

\section{Sequence Analysis}

Multiple sequence alignments were generated using PileUp of the GCG Wisconsin package (Version 10.0, Genetics Computer Group) and ClustalX (Thompson et al. 1997). Phylogenies were determined with PAUP* (D.L. Swofford, Smithsonian Institution) by bootstrap analysis using maximum parsimony (100 replicates) and neighbor-joining (1000 replicates; Saitou and Nei 1987). Maximum likelihood analysis was performed by quartet puzzling using TREE-PUZZLE 5.0 (Schmidt et al. 2002). Gene structure was analyzed using programs available at the NIX server (http://menu.hgmp.mrc. ac.uk/menu-bin/Nix). Pufferfish and zebrafish genome survey and trace sequences were obtained using the NCBI BLAST server (http://www.ncbi.nlm.nih.gov/BLAST). Zebulon nondegenerated consensus sequence (AY135221) was reconstructed by assembling trace sequences showing overlaps with $>95 \%$ nucleotide identity.

\section{ACKNOWLEDGMENTS}

We thank the Genoscope production teams (cloning, sequencing, and finishing), Corinne Cruaud (Genoscope) for sequencing assistance and Muriel Ronsin (Genoscope) for technical work. This work was supported by the French Museum National d'Histoire Naturelle, the Centre National de la Recherche Scientifique (CNRS) and the Ministère de la Recherche et de la Technologie (to MNHN and Genoscope), and by the BioFuture program of the German Ministry for Research and Education (BMBF) (to J.N.V.).

The publication costs of this article were defrayed in part by payment of page charges. This article must therefore be hereby marked "advertisement" in accordance with 18 USC section 1734 solely to indicate this fact.

\section{REFERENCES}

Altschul, S.F., Gish, W., Miller, W., Myers, E.W., and Lipman, D.J. 1990. Basic local alignment search tool. J. Mol. Biol. 215: $403-410$.

Aparicio, S., Chapman, J., Stupka, E., Putnam, N., Chia, J., Dehal, P., Christoffels, A., Rash, S., Hoon, S., Smit, A.F., et al. 2002. Whole-genome shotgun assembly and analysis of the genome of Fugu rubripes. Science 297: 1301-1310.

Arkhipova, I.R. and Morrison, H.G. 2001. Three retrotransposon families in the genome of Giardia lamblia: Two telomeric, one dead. Proc. Natl. Acad. Sci. 98: 14497-14502.

Bartolomé, C., Maside, X., and Charlesworth, B. 2002. On the abundance and distribution of transposable elements in the genome of Drosophila melanogaster. Mol. Biol. Evol. 19: 926-937.
Boeke, J.D. and Chapman, K.B. 1991. Retrotransposition mechanisms. Curr. Opin. Cell Biol. 3: 502-507.

Brenner, S., Elgar, G., Sandford, R., Macrae, A., Venkatesh, B., and Aparicio, S. 1993. Characterization of the pufferfish (Fugu) genome as a compact model vertebrate genome. Nature 366: $265-268$.

Burke, W.D., Malik, H.S., Rich, S.M., and Eickbush, T.H. 2002. Ancient lineages of non-LTR retrotransposons in the primitive eukaryote, Giardia lamblia. Mol. Biol. Evol. 19: 619-630.

Crollius, H.R., Jaillon, O., Dasilva, C., Ozouf-Costaz, C., Fizames, C., Fischer, C., Bouneau, L., Billault, A., Quetier, F., Saurin, W., et al. 2000. Characterization and repeat analysis of the compact genome of the freshwater pufferfish Tetraodon nigroviridis. Genome Res. 10: 939-949.

Danilevskaya, O.N., Arkhipova, I.R., Traverse, K.L., and Pardue, M.L. 1997. Promoting in tandem: The promoter for telomere transposon $H e T-A$ and implications for the evolution of retroviral LTRs. Cell 88: 647-655.

Dasilva, C., Hadji, H., Ozouf-Costaz, C., Nicaud, S., Jaillon, O., Weissenbach, J., and Crollius, H.R. 2002. Remarkable compartmentalization of transposable elements and pseudogenes in the heterochromatin of the Tetraodon nigroviridis genome. Proc. Natl. Acad. Sci. 99: 13636-13641.

Dimitri, P. and Junakovic, N. 1999. Revising the selfish DNA hypothesis. New evidence on accumulation of transposable elements in heterochromatin. Trends Genet. 15: 123-124.

Duvernell, D.D. and Turner, B.J. 1998. Swimmer 1, a new low-copy-number LINE family in teleost genomes with sequence similarity to mammalian L1. Mol. Biol. Evol. 15: 1791-1793.

Eickbush, T.H. and Furano, A.V. 2002. Fruit flies and humans respond differently to retrotransposons. Curr. Opin. Genet. Dev. 12: $669-674$.

Feng, Q., Moran, J.V., Kazazian Jr., H.H., and Boeke, J.D. 1996. Human $L 1$ retrotransposon encodes a conserved endonuclease required for retrotransposition. Cell 87: 905-916.

Fischer, C., Ozouf-Costaz, C., Roest Crollius, H., Dasilva, C., Jaillon, O., Bouneau, L., Bonillo, C., Weissenbach, J., and Bernot, A. 2000. Karyotype and chromosome location of characteristic tandem repeats in the pufferfish Tetraodon nigroviridis. Cytogenet. Cell Genet. 88: 50-55.

Fischer, C., Bouneau, L., Ozouf-Costaz, C., Crnogorac-Jurcevic, T., Weissenbach, J., and Bernot, A. 2002. Conservation of the T-cell receptor $\alpha / \delta$ linkage in the teleost fish Tetraodon nigroviridis. Genomics 79: 241-248.

Frame, I.G., Cutfield, J.F., and Poulter, R.T. 2001. New BEL-like LTR-retrotransposons in Fugu rubripes, Caenorhabditis elegans, and Drosophila melanogaster. Gene 263: 219-230.

Gabriel, A., Yen, T.J., Schwartz, D.C., Smith, C.L., Boeke, J.D., Sollner-Webb, B., and Cleveland, D.W. 1990. A rapidly rearranging retrotransposon within the miniexon gene locus of Crithidia fasciculata. Mol. Cell. Biol. 10: 615-624.

Gilbert, N., Lutz-Prigge, S., and Moran, J.V. 2002. Genomic deletions created upon LINE-1 retrotransposition. Cell 110: $315-325$.

Goodwin, T.J. and Poulter, R.T. 2001. The DIRS1 group of retrotransposons. Mol. Biol. Evol. 18: 2067-2082.

- 2002. A group of deuterostome Ty3/gypsy-like retrotransposons with Ty1/copia-like pol-domain orders. Mol. Genet. Genomics 267: 481-491.

Higashiyama, T., Noutoshi, Y., Fujie, M., and Yamada, T. 1997. Zepp, a LINE-like retrotransposon accumulated in the Chlorella telomeric region. $E M B O$ J. 16: 3715-3723.

International Human Genome Sequencing Consortium. 2001. Initial sequencing and analysis of the human genome. Nature 409: $860-921$.

Kidwell, M.G. 2002. Transposable elements and the evolution of genome size in eukaryotes. Genetica 115: 49-63.

Lyozin, G.T., Makarova, K.S., Velikodvorskaja, W., Zelentsova, H.S., Khechumian, R.R., Kidwell, M.G., Koonin, E.V., and Evgen'ev, M.B. 2001. The structure and evolution of Penelope in the virilis species group of Drosophila: An ancient lineage of retroelements. J. Mol. Evol. 52: 445-456.

Malik, H.S. and Eickbush, T.H. 1999. Modular evolution of the integrase domain in the Ty3/Gypsy class of LTR retrotransposons. J. Virol. 73: 5186-5190.

. 2000. NeSL-1, an ancient lineage of site-specific non-LTR retrotransposons from Caenorhabditis elegans. Genetics 154: 193-203.

Malik, H.S., Burke, W.D., and Eickbush, T.H. 1999. The age and evolution of non-LTR retrotransposable elements. Mol. Biol. Evol. 16: $793-805$.

\section{Genome Research}


Pardue, M.L. and Debaryshe, P.G. 2000. Drosophila telomere transposons: Genetically active elements in heterochromatin. Genetica 109: 45-52.

Poulter, R. and Butler, M. 1998. A retrotransposon family from the pufferfish (fugu) Fugu rubripes. Gene 215: 241-249.

Poulter, R., Butler, M., and Ormandy, J. 1999. A LINE element from the pufferfish (fugu) Fugu rubripes which shows similarity to the CR1 family of non-LTR retrotransposons. Gene 227: 169-179.

Roest Crollius, H., Jaillon, O., Bernot, A., Dasilva, C., Bouneau, L., Fischer, C., Fizames, C., Wincker, P., Brottier, P., Quetier, F., et al. 2000. Estimate of human gene number provided by genome-wide analysis using Tetraodon nigroviridis DNA sequence. Nat. Genet. 25: 235-238.

Saitou, N. and Nei, M. 1987. The neighbor-joining method: A new method for reconstructing phylogenetic trees. Mol. Biol. Evol. 4: $406-425$.

Schmidt, H.A., Strimmer, K., Vingron, M., and von Haeseler, A. 2002. TREE-PUZZLE: Maximum likelihood phylogenetic analysis using quartets and parallel computing. Bioinformatics 18: $502-504$.

Symer, D.E., Connelly, C., Szak, S.T., Caputo, E.M., Cost, G.J., Parmigiani, G., and Boeke, J.D. 2002. Human L1 retrotransposition is associated with genetic instability in vivo. Cell 110: 327-338.

Takahashi, H., Okazaki, S., and Fujiwara, H. 1997. A new family of site-specific retrotransposons, SART1, is inserted into telomeric repeats of the silkworm, Bombyx mori. Nucleic Acids Res. 25: $1578-1584$.

Thompson, J.D., Gibson, T.J., Plewniak, F., Jeanmougin, F., and Higgins, D.G. 1997. The ClustalX windows interface: Flexible strategies for multiple sequence alignment aided by quality analysis tools. Nucleic Acids Res. 24: 4876-4882.

Vaury, C., Bucheton, A., and Pelisson, A. 1989. The $\beta$ heterochromatic sequences flanking the $I$ elements are themselves defective transposable elements. Chromosoma 98: $215-224$.

Volff, J.-N., Körting, C., Sweeney, K., and Schartl, M. 1999. The non-LTR retrotransposon Rex3 from the fish Xiphophorus is widespread among teleosts. Mol. Biol. Evol. 16: 1427-1438.

Volff, J.-N., Körting, C., and Schartl, M. 2000. Multiple lineages of the non-LTR retrotransposon Rex1 with varying success in invading fish genomes. Mol. Biol. Evol. 17: 1673-1684.

Volff, J.-N., Hornung, U., and Schartl, M. 2001a. Fish retroposons related to the Penelope element of Drosophila virilis define a new group of retrotransposable elements. Mol. Genet. Genomics 265: $711-720$

Volff, J.-N., Körting, C., Altschmied, J., Duschl, J., Sweeney, K., Wichert, K., Froschauer, A., and Schartl, M. 2001b. Jule from the fish Xiphophorus is the first complete vertebrate Ty3/Gypsy retrotransposon from the Mag family. Mol. Biol. Evol. 18: $101-111$

Volff, J.-N., Körting, C., Froschauer, A., Sweeney, K., and Schartl, M. 2001c. Non-LTR retrotransposons encoding a restriction enzyme-like endonuclease in vertebrates. J. Mol. Evol. 52: 351-360.

Volff, J.-N., Körting, C., Meyer, A., and Schartl, M. 2001d. Evolution and discontinuous distribution of Rex 3 retrotransposons in fish. Mol. Biol. Evol. 18: 427-431.

Volff, J.-N., Körting, C., and Schartl, M. 2001e. Ty3/Gypsy retrotransposon fossils in mammalian genomes: Did they evolve into new cellular functions? Mol. Biol. Evol. 18: 266-270.

Xiong, Y. and Eickbush, T.H. 1990. Origin and evolution of retroelements based upon their reverse transcriptase sequences. EMBO J. 9: 3353-3362.

Yang, J., Malik, H.S., and Eickbush, T.H. 1999. Identification of the endonuclease domain encoded by $R 2$ and other site-specific, non-long terminal repeat retrotransposable elements. Proc. Natl. Acad. Sci. 96: 7847-7852.

\section{WEB SITE REFERENCES}

http://fugu.hgmp.mrc.ac.uk/; The Fugu Genomics site at the UK HGMP Resource Centre.

http://menu.hgmp.mrc.ac.uk/menu-bin/Nix; The Bio-informatics Application Server at the UK HGMP Resource Centre.

http://www.ensembl.org/Danio_rerio/blastview; The Zebrafish BLAST server at the Wellcome Trust Sanger Institute.

http://www.ncbi.nlm.nih.gov/BLAST; The BLAST server at the National Center for Biotechnology Information.

http://www.ncbi.nlm.nih.gov/blast/tracemb.html; The Trace server at the National Center for Biotechnology information.

Received August 21, 2002; accepted in revised form April 18, 2003.
Genome Research www.genome.org 


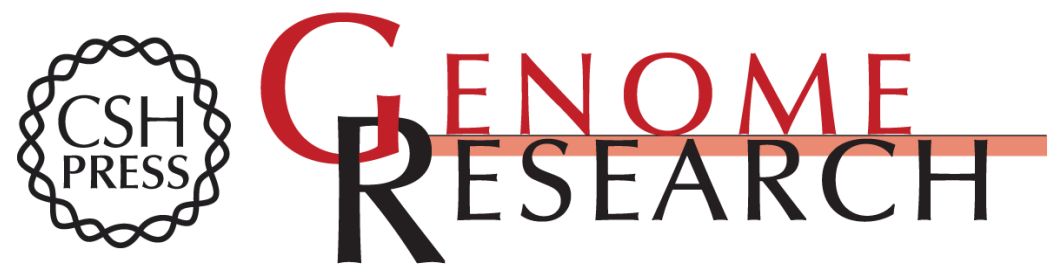

\section{An Active Non-LTR Retrotransposon With Tandem Structure in the Compact Genome of the Pufferfish Tetraodon nigroviridis}

Laurence Bouneau, Cécile Fischer, Catherine Ozouf-Costaz, et al.

Genome Res. 2003 13: 1686-1695

Access the most recent version at doi:10.1101/gr.726003

References This article cites 46 articles, 9 of which can be accessed free at:

http://genome.cshlp.org/content/13/7/1686.full.html\#ref-list-1

\section{License}

Email Alerting Receive free email alerts when new articles cite this article - sign up in the box at the Service top right corner of the article or click here.

\section{Affordable, Accurate Sequencing.}

